9. S. G. Krein, and O. I. Prozorovskaya, An analogue of Seidel's method for operator equations, Voronez. Gos. Univ., Trudy Sem. Functional. Anal. 5 (1957), 35-38.

10. W. V. Petryshyn, The generalized overrelaxation method for the approximate solution of operator equations in Hilbert space, J. Soc. Indust. Appl. Math. 10 (1962), 675-690.

11. S. Schechter, Relaxation methods for linear equations, Comm. Pure Appl. Math. 12 (1959), 313-335.

12. R. S. Varga, A comparison of the successive overrelaxation method and semiiterative methods using Chebyshev polynomials, J. Soc. Indust. Appl. Math. 5 (1957), 39-46.

13. D. Young, Iterative methods for solving partial difference equations of elliptic type, Trans. Amer. Math. Soc. 76 (1954), 91-111.

NeW YoRK UNIVERSITY

\title{
NOTE ON A NONLINEAR VOLTERRA EQUATION
}

\section{J. J. LEVIN ${ }^{1}$ AND J. A. NOHEL 2}

1. Introduction. We investigate the solutions of

$$
x^{\prime}(t)=-\int_{0}^{t} a(t-\tau) g(x(\tau)) d \tau \quad\left(,=\frac{d}{d t}\right)
$$

as $t \rightarrow \infty$, where $a(t)$ is completely monotonic on $0 \leqq t<\infty$ and where $g(x)$ is a (nonlinear) spring. Under this hypothesis, (1.1) was shown in [2] to be relevant to certain physical applications and results were obtained there for the linear case $g(x) \equiv x$. (If $a(t) \equiv a(0)$, then (1.1) reduces to the nonlinear oscillator $x^{\prime \prime}+a(0) g(x)=0$.) Equation (1.1) was studied in [1] under less hypothesis on $a(t)$. However, while the result is weaker than that of [1], the present approach draws together such different notions of positivity as Liapounov functions, completely monotonic functions, and kernels of positive type. It also provides a new Liapounov function for (1.1). Specifically, we prove the

ThEOREM. Let $a(t)$ and $g(x)$ satisfy

$$
\begin{array}{r}
a(t) \in C[0, \infty),(-1)^{k} a^{(k)}(t) \geqq 0(0<t<\infty ; k=0,1,2, \cdots), \\
g(x) \in C(-\infty, \infty), x g(x)>0(x \neq 0), G(x)=\int_{0}^{x} g(\xi) d \xi \rightarrow \infty \\
(|x| \rightarrow \infty) .
\end{array}
$$
1962.

Presented to the Society, January 24,1963 ; received by the editors September 6 ,

${ }^{1}$ MIT Lincoln Laboratory. Operated with support from the U. S. Army, Navy, and Air Force.

2 Partially supported by the National Science Foundation (G-19925). 
If $a(t) \not \equiv a(0)$ and if $u(t)$ is any solution of (1.1) which exists on $0 \leqq t<\infty$, then

$$
\lim _{t \rightarrow \infty} u^{(j)}(t)=0 \quad(j=0,1,2) .
$$

In [1] only $k=0,1,2,3$ is required in the analogue of (1.2), rather than complete monotonicity. The Liapounov function used there was

$$
\begin{aligned}
E(t)= & G(u(t))+\frac{1}{2} a(t)\left[\int_{0}^{t} g(u(\tau)) d \tau\right]^{2} \\
& -\frac{1}{2} \int_{0}^{t} a^{\prime}(t-\tau)\left[\int_{\tau}^{t} g(u(s)) d s\right]^{2} d \tau \geqq 0 .
\end{aligned}
$$

In (1.5) and the sequel $u(t)$ is the solution of (1.1) on $0 \leqq t<\infty$ being considered. Remarks concerning existence, uniqueness, as well as background information and references, may be found in [1].

2. Positivity. In this section we motivate the hypothesis (1.2) and also obtain the Liapounov function. Suppose that the origin of the problem is such that $a(t) \geqq 0, a(t) \in C[0, \infty), a^{\prime}(t) \in L_{1}(0, T)$ for each $0<T<\infty$, and (1.3) are all required. Then clearly

$$
V(t)=G(u(t))+\frac{1}{2} \int_{0}^{t} \int_{0}^{t} a(\tau+s) g(u(t-\tau)) g(u(t-s)) d \tau d s
$$

is nonnegative if the second term is. (From (1.1) and (1.3), $V(t)$ may be interpreted as the sum of a potential and kinetic energy.)

The second (or kinetic) term of $V(t)$ will be nonnegative if it is assumed that $a(\tau+s)$ is a kernel of positive type $[3$, p. 270] on the square $0<\tau, s<t$ for each $0<t<\infty$. By a theorem of Boas and Widder $[3$, pp. 273-275], this will be the case if and only if

$$
a(t)=\int_{-\infty}^{\infty} \exp [-\xi t] d \alpha(\xi) \quad(0<t<\infty),
$$

where $\alpha(\xi)$ is nondecreasing on $-\infty<\xi<\infty$ (and may be assumed normalized: $\left.\alpha(0)=0, \alpha(\xi)=\frac{1}{2}[\alpha(\xi+)+\alpha(\xi-)]\right)$.

Differentiating (2.1) yields

$$
V^{\prime}(t)=\int_{0}^{t} \int_{0}^{t} a^{\prime}(\tau+s) g(u(t-\tau)) g(u(t-s)) d \tau d s,
$$

where we have used the above assumptions, (1.1), the absolutely continuity of $a(t)$ on $0<\epsilon \leqq t \leqq T<\infty$ implied by (2.2), and an obvious change of variables. If $V(t)$ is to serve as a Liapounov function for 
(1.1), then $V^{\prime}(t)$ must be nonpositive. By (2.3) the latter will be assured if $-a^{\prime}(\tau+s)$ is a kernel of positive type on the square $0<\tau$, $s<t$ for each $0<t<\infty$. However, this is compatible with (2.2) if and only if $\alpha(-\infty)=\alpha(0-)$. Thus, also using $a(t) \in C[0, \infty)$, one has

$$
a(t)=\int_{0}^{\infty} \exp [-\xi t] d \alpha(\xi) \quad(0 \leqq t<\infty)
$$

where $\alpha(\infty)<\infty$. By a theorem of S. Bernstein [3, p. 160], (1.2) and (2.4) are equivalent.

Having motivated the hypothesis (1.2) and obtained a Liapounov function $V(t)$, it is interesting to compare the two Liapounov functions (1.5) and (2.1). It is obvious that if $a(t) \equiv a(0)$, then $E(t)$ $\equiv V(t) \quad(0 \leqq t<\infty)$. We now prove a strong form of the converse statement. In particular, if $u(0) \neq 0$ and if $E(t) \equiv V(t)\left(0 \leqq t \leqq t_{0}\right)$ for some $0<t_{0}<\infty$, then $a(t) \equiv a(0)(0 \leqq t<\infty)$. For this one need only assume that $a(t) \in C[0, \infty),(-1)^{k} a^{(k)}(t) \geqq 0(0<t<\infty ; k=0,1,2)$, $g(x) \in C(-\infty, \infty)$, and $g(x)=0$ implies $x=0$. Direct calculations show that (1.5) and (2.1) may also be written as

$$
\begin{aligned}
& E(t)=G(u(t))+\int_{0}^{t} g(u(\tau))\left\{\int_{\tau}^{t} a(t-\tau) g(u(s)) d s\right\} d \tau, \\
& V(t)=G(u(t))+\int_{0}^{t} g(u(\tau))\left\{\int_{\tau}^{t} a(2 t-\tau-s) g(u(s)) d s\right\} d \tau .
\end{aligned}
$$

Hence

$$
\int_{0}^{t} g(u(\tau))\left\{\int_{\tau}^{t}[a(t-\tau)-a(2 t-\tau-s)] g(u(s)) d s\right\} d \tau \equiv 0
$$

$$
\left(0 \leqq t \leqq t_{0}\right)
$$

From the hypothesis on $a(t)$ one has $a(t-\tau)-a(2 t-\tau-s) \geqq 0$ for $0 \leqq \tau, s \leqq t$. As $u(0) \neq 0$, there exists by continuity and the hypothesis on $g(x)$ a $0<t_{1} \leqq t_{0}$ such that $g(u(t)) \neq 0$ for $0 \leqq t \leqq t_{1}$. It is now clear from (2.5) that $a(t-\tau) \equiv a(2 t-\tau-s)\left(0 \leqq \tau \leqq s \leqq t \leqq t_{1}\right)$. The latter together with $a(t) \in C[0, \infty)$ easily implies $a(t) \equiv a(0) \quad\left(0 \leqq t \leqq 2 t_{1}\right)$. Hence $a^{\prime}(t) \equiv 0\left(0 \leqq t \leqq 2 t_{1}\right)$. However, as $-a^{\prime}(t), a^{\prime \prime}(t) \geqq 0$ it follows that $a^{\prime}(t) \equiv 0(0 \leqq t<\infty)$ so that $a(t) \equiv a(0) \quad(0 \leqq t<\infty)$, which proves the assertion.

3. Proof of the Theorem. Let $a(t), V(t)$ be given by (2.4), (2.1) respectively. Define 


$$
\Gamma(\xi, t)=\int_{0}^{t} \exp [-\xi(t-\tau)] g(u(\tau)) d \tau \quad(0 \leqq \xi, t<\infty) .
$$

Then

$$
\frac{\partial \Gamma}{\partial t}(\xi, t)=g(u(t))-\xi \Gamma(\xi, t) \quad(0 \leqq \xi, t<\infty) .
$$

Clearly $\Gamma(\xi, t), \Gamma_{t}(\xi, t)$ are bounded functions of $\xi$ on $0 \leqq \xi<\infty$ for each fixed $t$ in $0 \leqq t<\infty$. Hence, by Fubini's theorem, one may write (1.1) (with $u(t)$ replacing $x(t)),(2.1)$ as

$$
\begin{array}{ll}
u^{\prime}(t)=-\int_{0}^{\infty} \Gamma(\xi, t) d \alpha(\xi) & (0 \leqq t<\infty), \\
V(t)=G(u(t))+\frac{1}{2} \int_{0}^{\infty} \Gamma^{2}(\xi, t) d \alpha(\xi) \geqq 0 & (0 \leqq t<\infty)
\end{array}
$$

respectively and, moreover,

$$
V^{\prime}(t)=-\int_{0}^{\infty} \xi \Gamma^{2}(\xi, t) d \alpha(\xi) \leqq 0
$$$$
(0 \leqq t<\infty)
$$

From (3.4), (3.5) one has

$$
G(u(t)) \leqq V(t) \leqq V(0)=G\left(u_{0}\right) \quad(0 \leqq t<\infty),
$$

where $u_{0}=u(0)$. It follows from (1.3) that

$$
|u(t)| \leqq K<\infty \quad(0 \leqq t<\infty) .
$$

In (3.6) and subsequent formulas $K=K\left(u_{0}\right)<\infty$, where $K$ may vary from formula to formula, and $K\left(u_{0}\right) \rightarrow 0$ as $u_{0} \rightarrow 0$. Thus

(3.7) $|\Gamma(\xi, t)| \leqq K t,|\xi \Gamma(\xi, t)| \leqq K,\left|\Gamma_{t}(\xi, t)\right| \leqq K(0 \leqq \xi, t<\infty)$.

Differentiating (3.3) (using Fubini's theorem) yields

$$
u^{\prime \prime}(t)=-g(u(t)) \alpha(\infty)+\int_{0}^{\infty} \xi \Gamma(\xi, t) d \alpha(\xi) \quad(0 \leqq t<\infty),
$$

which together with (1.3), (3.6), and (3.7) implies

$$
\left|u^{\prime \prime}(t)\right| \leqq K
$$$$
(0 \leqq t<\infty)
$$

By (3.6), (3.9), and the mean value theorem one has

$$
\left|u^{\prime}(t)\right| \leqq K \quad(0 \leqq t<\infty) .
$$

From (3.2), (3.5) there results 


$$
V^{\prime \prime}(t)=-2 g(u(t)) \int_{0}^{\infty} \xi \Gamma(\xi, t) d \alpha(\xi)+2 \int_{0}^{\infty} \xi^{2} \Gamma^{2}(\xi, t) d \alpha(\xi)
$$

$$
(0 \leqq t<\infty) \text {, }
$$

so that by (1.3), (3.6), (3.7) one has $\left|V^{\prime \prime}(t)\right| \leqq K$ on $0 \leqq t<\infty$. The latter together with (3.4), (3.5), and the mean value theorem implies (see Lemma 1 of [1])

$$
\lim _{t \rightarrow \infty} V^{\prime}(t)=-\lim _{t \rightarrow \infty} \int_{0}^{\infty} \xi \Gamma^{2}(\xi, t) d \alpha(\xi)=0 .
$$

We assert that there exists a $\xi_{1}>0$ such that $\Gamma\left(\xi_{1}, t\right) \rightarrow 0$ as $t \rightarrow \infty$. Suppose not and let $0<\xi_{0}<\infty$. Then there exist a $\lambda=\lambda\left(\xi_{0}\right)>0$ and a sequence $\left\{t_{n}=t_{n}\left(\xi_{0}\right)\right\}$, where $t_{n} \rightarrow \infty$ as $n \rightarrow \infty$, such that $\left|\Gamma\left(\xi_{0}, t_{n}\right)\right|$ $\geqq \lambda$. From (3.1) one has

$$
\frac{\partial \Gamma}{\partial \xi}(\xi, t)=-\int_{0}^{t} \exp [-\xi(t-\tau)](t-\tau) g(u(\tau)) d \tau \quad(0 \leqq \xi, t<\infty) .
$$

Let $\delta=\min \left(\xi_{0} / 2, \lambda \xi_{0}^{2} / 8 K_{1}\right), I_{\xi_{0}}=\left\{\xi|| \xi-\xi_{0} \mid \leqq \delta\right\}$, where $|g(u(t))|$ $\leqq K_{1}$ on $0 \leqq t<\infty$. Then by the mean value theorem and (3.12) one obtains $\left|\Gamma\left(\xi, t_{n}\right)-\Gamma\left(\xi_{0}, t_{n}\right)\right| \leqq \lambda / 2\left(\xi \in I_{\xi_{0}}\right)$ so that, as $\left|\Gamma\left(\xi_{0}, t_{n}\right)\right| \geqq \lambda$, $\left|\Gamma\left(\xi, t_{n}\right)\right| \geqq \lambda / 2\left(\xi \in I_{\xi_{0}}\right)$. Hence

$$
\begin{aligned}
\int_{0}^{\infty} \xi \Gamma^{2}\left(\xi, t_{n}\right) d \alpha(\xi) & \geqq \int_{I_{\xi_{0}}} \xi \Gamma^{2}\left(\xi, t_{n}\right) d \alpha(\xi) \\
& \geqq \frac{\lambda^{2} \xi_{0}}{8}\left[\alpha\left(\xi_{0}+\delta\right)-\alpha\left(\xi_{0}-\delta\right)\right] \geqq 0,
\end{aligned}
$$

which with (3.11) yields $\alpha\left(\xi_{0}+\delta\right)=\alpha\left(\xi_{0}-\delta\right)$. As this is true for each $0<\xi_{0}<\infty$, it follows that $\alpha(0+)=\alpha(\infty)$ which contradicts $a(t) \not \equiv a(0)$. Thus, there exists a $\xi_{1}>0$ with the asserted property.

Let $f(t)=\exp \left[-\xi_{1} t\right], p(t)=g(u(t))$ for $0 \leqq t<\infty$ and $f(t)=p(t) \equiv 0$ for $-\infty<t<0$. From the preceding paragraph one has

$$
\lim _{t \rightarrow \infty} \Gamma\left(\xi_{1}, t\right)=\lim _{t \rightarrow \infty} \int_{-\infty}^{\infty} f(t-\tau) p(\tau) d \tau=0 .
$$

By applying Pitt's form of Wiener's tauberian theorem [3, p. 211] to (3.13), we now show that $p(t) \rightarrow 0$ as $t \rightarrow \infty$. (A longer elementary argument could also be used here.) Clearly, $f \in L_{1}(-\infty, \infty)$ and its Fourier transform $\hat{f}(s)=(2 \pi)^{-1 / 2}\left(\xi_{1}+i s\right)^{-1} \neq 0$ for $-\infty<s<\infty$. As $|p(t)| \leqq K(-\infty<t<\infty)$, there remains only to show that $p(t)$ is a slowly decreasing function in $(-\infty, \infty)$. For this it suffices to show 
that $p\left(t_{i}\right)-p\left(s_{i}\right) \rightarrow 0$ as $i \rightarrow \infty$ if $\left\{t_{i}\right\},\left\{s_{i}\right\}$ are any sequences satisfying $t_{i}>s_{i}>0$ and $s_{i} \rightarrow \infty, t_{i}-s_{i} \rightarrow 0$ as $i \rightarrow \infty$. However, from (1.3), (3.6), (3.10), the mean value theorem, and uniform continuity, it is clear that $g\left(u\left(t_{i}\right)\right)-g\left(u\left(s_{i}\right)\right) \rightarrow 0$ as $i \rightarrow \infty$ for such sequences. Thus, $p(t) \rightarrow 0$ as $t \rightarrow \infty$, which together with (1.3) and (3.6) yields $u(t) \rightarrow 0$ as $t \rightarrow \infty$ (i.e., $(1.4, j=0))$.

From $(1.4, j=0)$ and (3.9) one has $(1.4, j=1)$ by the mean value theorem. From $(1.4, j=0)$ and (3.1) it is an elementary exercise to show that $\xi \Gamma(\xi, t) \rightarrow 0$ as $t \rightarrow \infty$ uniformly with respect to $\xi$ on $0 \leqq \xi<\infty$. This together with $(1.4, j=0),(1.3)$, and (3.8) implies $(1.4, j=2)$, which completes the proof.

\section{REFERENCES}

1. J. J. Levin, The asymptotic behavior of the solution of a Volterra equation, Proc. Amer. Math. Soc. 14 (1963), 534-541.

2. J. J. Levin, and J. A. Nohel, On a system of integrodifferential equations occurring in reactor dynamics, J. Math. Mech. 9 (1960), 347-368.

3. D. V. Widder, The Laplace transform, Princeton Univ. Press, Princeton, N. J., 1946.

LINCOLN LABORATORY AND

UNIVERSITY OF WISCONSIN 